\title{
Serial nominations for the AWH initiative: The paradigm of seven-stone antas and beyond
}

\author{
Juan Antonio Belmonte ${ }^{1}$, César González García ${ }^{2}$ \\ and Michael Hoskin ${ }^{3}$ \\ ${ }^{1}$ Instituto de Astrofísica de Canarias, Spain \\ email: jba@iac.es \\ ${ }^{2}$ Incipit, CSIC, Santiago de Compostela, Spain \\ email: cesar.gonzalez-garcia@incipit.csic.es \\ ${ }^{3}$ Cambridge University, United Kingdom \\ email: michael.hoskin@ntlworld.com
}

\begin{abstract}
In this short report we examine the ideal status of the seven-stone antas (a type of very ancient megalithic monument in the southwest of the Iberian Peninsula) as an excellent candidate for a serial nomination within the Astronomy and World Heritage Initiative. This case will be compared with an extraordinary set of dolmens at the other side of the Mediterranean, within the Transjordan Plateau, worthy of being protected under the umbrella of the same initiative but which are in serious danger of 'extinction'.
\end{abstract}

\section{Seven-stone antas}

Seven-stone antas are a group of megalithic monuments that were built in the southwest of the Iberian Peninsula for a period of some one thousand years in the 4th millennium B.C. - and possibly earlier - for the burial of people presumably belonging to a pastoral culture. They can be described as standard corridor dolmens but their most impressive fact is that they were constructed with a surprisingly consistent architectural concept over an extended period of time (hence their common name) and with a pattern of orientations that certainly situate them among the oldest monuments on Earth with indisputable astronomical orientations (Hoskin 2001). They were built in an extended area of the present day regions of Alentejo, in Portugal, where most of the antas are located, and Extremadura, in Spain (see Fig. 1). The presence of such impressive, extremely old monuments at both sides of the Spanish-Portuguese frontier and their reliability as an extremely important Case Study for the UNESCO Astronomical Heritage initiative makes of this particular set of megalithic tombs an extraordinary opportunity as a paradigm for serial nominations (Belmonte et al. 2015).

The numbers of these monuments is around 200 but somewhat fewer are in a sufficiently good state of preservation that alignment studies can be performed. The area occupied by the antas is so huge that it is evident that a full protection of all the monuments would be an extremely difficult task. However, we strongly believe that seven-stone antas as a group, and especially a particularly well-preserved set of them in the areas of Valencia de Alcántara (Spain) and central Alentejo (Portugal), deserve recognition within the framework of the Astronomy and World Heritage Initiative. This is supported not only by the fact that they represent the oldest group of monuments in the world with an unmistakable astronomical interest in their orientation - and presumably an interest in 


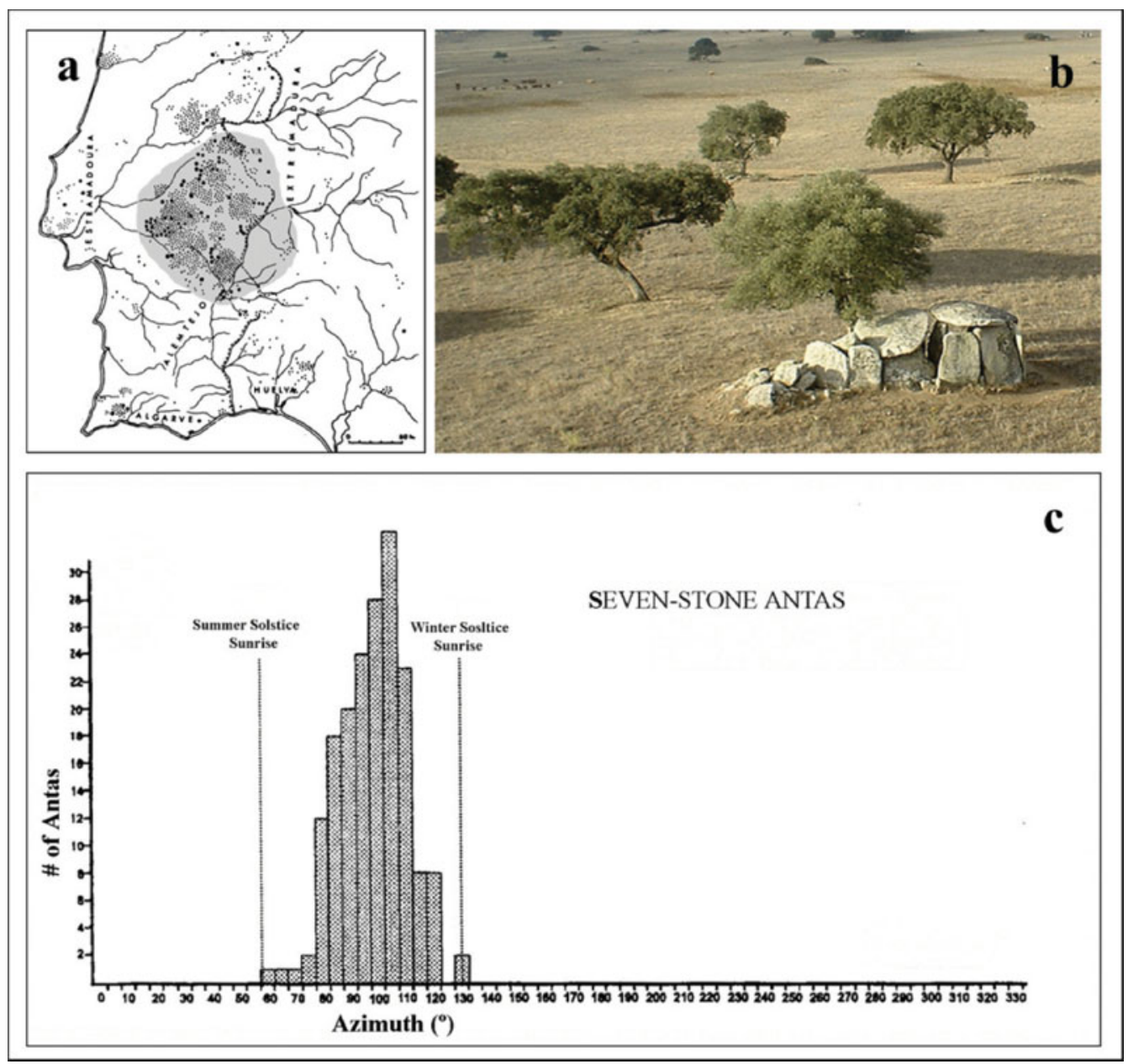

Figure 1. Seven-stone antas: area of distribution (a), together with a well-preserved example in the Portuguese Alentejo (b) and a histogram of orientation of a set of 172 measurable monuments. Diagram by the authors.

the sky as the motivation for this aspect of their construction - but also because they extend over a vast geographical area within both of the modern Iberian states: Spain and Portugal. This would certainly serve to guarantee the protection of the sites from any possible challenge in the future and for gaining a deserved recognition among modern society.

\section{Dolmens in Jordan}

Interestingly, at the other end of the Mediterranean, on the plateau to the east of the Jordan River valley, exist some of the largest and most attractive groups of dolmens of the Levant, which are fully comparable to the best exemplars to the west (see Fig. 2). The orientations of more than 180 dolmens have been measured in the field in Jordan, permitting the study of the astronomical aspects of the megalithic phenomenon in the region (Belmonte et al. 2013). The evidence shows that Early Bronze Age populations of the Transjordan plateau also orientated their tombs according to their celestial 


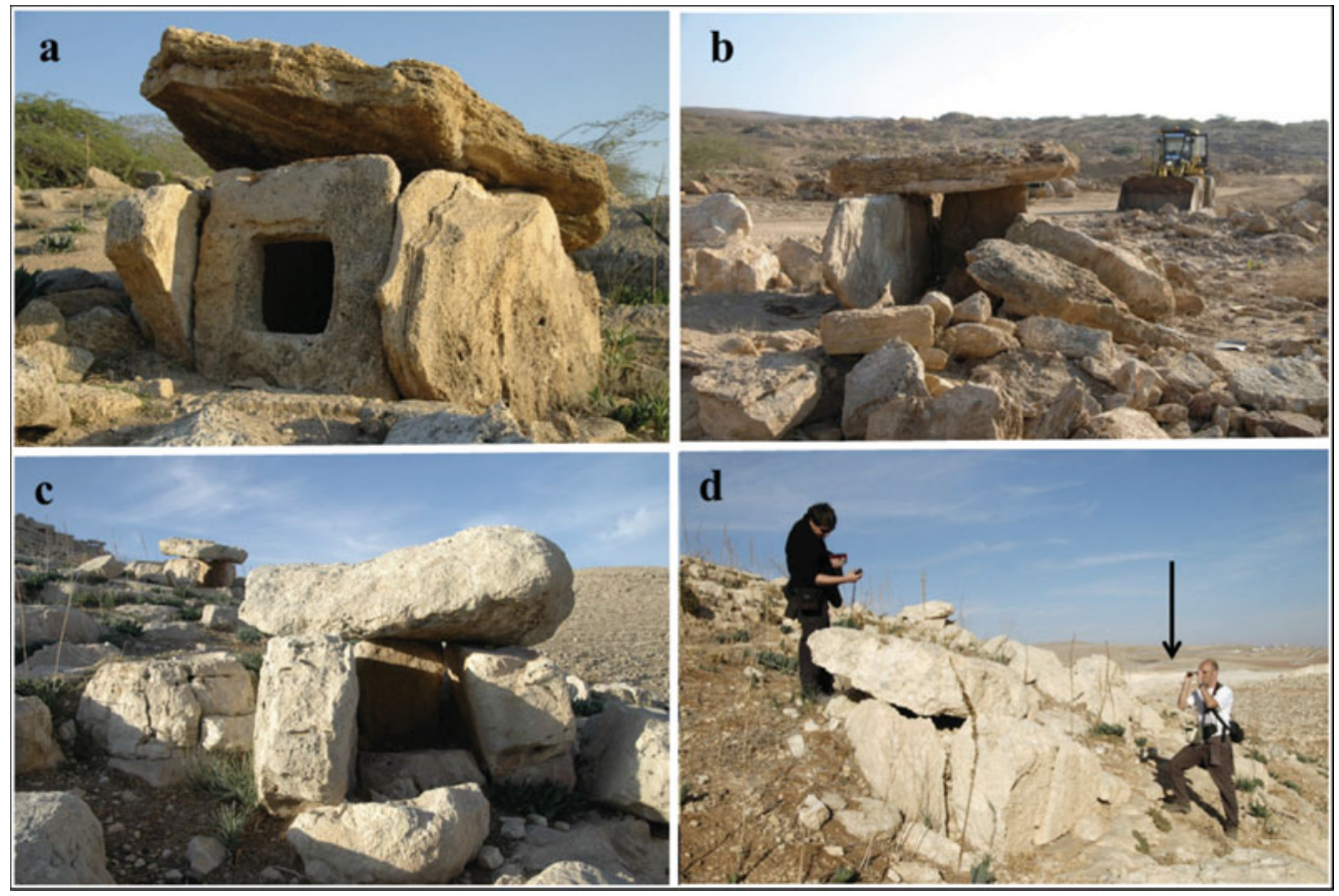

Figure 2. Two examples of endangered megalithic necropolises in Jordan: a nicely preserved dolmen and another on the way to destruction at Ala Safat (a) and the huge necropolis at Al Murayghat (b) where a gigantic quarry is steadily approaching the site and may engulf it within a few years if nothing prevents it. Diagram by the authors.

connections (but also, to a minor extent, according to the terrestrial landscape), providing a second potential case for serial nomination. However, in contrast to the Iberian Peninsula, it is especially troubling that specialists in the Levant are now faced with the rapid destruction of the Hashemite Kingdom of Jordan's megalithic heritage owing to the huge quarries that are rapidly expanding and, more generally, to the fast urban development in the country (see Fig. 2). It is indeed necessary to raise awareness through UNESCO promotion of these beautiful and extremely interesting monuments - especially among the local people, perhaps the most difficult ones to persuade - so that they may be seriously protected as soon as possible by local authorities before it is too late. Otherwise, we might be facing the loss of a relevant component not only of archaeological, but also of astronomical, world heritage.

\section{References}

Belmonte, J. A., González García, A. C. \& Polcaro, A. 2013, J. Hist. Ast., 44, 429

Belmonte, J. A., Tirapicos, L., \& Ruggles, C. 2015, in: C. Ruggles \& M. Cotte (eds.), Heritage sites of Astronomy and Archaeoastronomy in the Context of the UNESCO World Heritage Convention: Volume II (Bognor Regis: Ocarina Books), in press

Hoskin, M. 2001, Tombs, Temples and their Orientations : a New Perspective on Mediterranean Prehistory (Bognor Regis: Ocarina Books) 